\title{
Influence of Sheet Conditions on In-Plane Strain Evolution via Ex-Situ Tensile Deformation of Ti-3Al-2.5V at Room Temperature
}

\author{
J.S Kwame ${ }^{1}$, E. Yakushina ${ }^{2}$, P. Blackwell ${ }^{1,2}$ \\ 1. Department of Design, Manufacturing and Engineering Management, University of Strathclyde, United \\ Kingdom \\ 2. Advanced Forming Research Centre (AFRC), University of Strathclyde, United Kingdom \\ E-mail: james.kwame@strath.ac.uk
}

Received: 2 September 2020; Accepted: 6 October 2020; Available online: 10 November 2020

\begin{abstract}
Localised plastic deformation evolution was examined in a near alpha Ti-3Al-2.5V alloy with indent defect and defect free surfaces using digital image correlation, an interrupted uniaxial tensile test and scanning electron microscopy. The main aim was to understand the role of the localised strain evolution at micro scale and the underlying deformation mechanisms that influence the global mechanical behaviour of the material. The microstructures captured at different stages of deformation were processed using a digital image correlation system, whose outputs were analysed through Matlab, to ascertain the localised strain evolution observed in each surface condition. This work found that the strains observed at the deformation bands along the indent defect edge, were significantly higher than those observed in the deformed $\beta$ phase field. The deformation bands concentrating at the tip of the indent defect acted as a fertile site for early crack nucleation and propagation with a reduced localised fracture strain. For a defect free surface, the absence of defect zones acting as a high stress concentration site meant that strain aggregation was minimised and the $\alpha$ phase field was able to sufficiently accommodate the $\beta$ phase deformation resulting in higher fracture strains.
\end{abstract}

Keywords: Titanium; Surface conditions; Localised strain; Deformation bands; Plastic deformation; Digital image correlation.

\section{Introduction}

Titanium and its alloys have gained wide recognition across the aerospace industry due to their excellent properties including their low density compared to steels, good high temperature properties and enhanced corrosion resistance and compatibility with carbon fibre composites as compared to aluminium [1]. The nature of the properties of titanium and its alloys means that they are employed in other applications for which their integrity in terms of damage resistance, fatigue properties and toughness are of the essence.

Sheet metals employed in the manufacture of components are often prone to accumulating surface inhomogeneity in the form of indents, scratches and notches. These are often picked up during transport, or as a result of handling within a production facility. Considering the notch sensitivity of titanium [2], there is the need to examine if the presence of surface inhomogeneities influence the localised deformation mode of titanium alloys. Generally, the overall deformation mode of a material governs its formability. The role deformation modes play during the tensile deformation of titanium alloys are linked to a variety of factors. Li et al., [3] compared the deformation behaviour of pure titanium, and Ti-5Al-2.5Sn which is a near alpha alloy. They found that prismatic slip was the most dominant deformation mode in both materials with basal slip activity quite strong in Ti-5Al2.5Sn. Lin et al., [4] working on two phased Ti-6Al-2Nb-1Ta- $0.8 \mathrm{Mo} \alpha+\beta$ alloy suggested that heat treating the original Widmanstätten microstructure to give a different range of microstructures including; $\alpha+\beta$, tempered martensite, coarse and fine Widmanstätten, resulted in different deformation modes. Their work attributed the differences in deformation mode to the presence of Widmanstätten colony boundaries, which served as an obstacle to slip. Li et al., [5] studied deformation behaviour in Ti-6Al-4V in tension at 296K and 728K. At 296K the authors found that prismatic slip dominated, with basal slip making a secondary contribution. However, with temperature rise basal slip became more common and the relative roles of prismatic and basal slip were reversed at $728 \mathrm{~K}$. Li et al., found that variations in the deformation mode in Ti-5Al-2.5Sn were attributable to the influence of alloying constituents [6]. The authors showed that, basal and prismatic slip are dominant at $296 \mathrm{~K}$ and $728 \mathrm{~K}$ during tensile deformation of the alloy, with twinning drastically reduced at $728 \mathrm{~K}$ due to the presence of the aluminium. Fitzner et al., [7] in their work on binary alpha titanium aluminide alloys also reported that, increasing the aluminium content increases twinning with the dominant type observed to be $\{10-12\}<10-11>$ tensile twinning. 
Texture is reported to play a major role in titanium deformation mode evolution. Lainé and Knowles [8] in their studies on deformation twinning of pure titanium attributed the unusual occurrence of $\{11-24\}$ twinning deformation observed in the coarse grains to the material texture. Li et al., [9] utilising electron backscatter based trace analysis on Ti-3Al-2.5V found that prismatic slip was the dominant deformation mode. Their work examined the behaviour of plates with two different textures, those performed under tensile deformation at 296K and 728K (plate 1) as well as those studied under tensile creep at 728K (plate 2). Prismatic slip dominated in both plates, although the level of prismatic slip was found to be influenced by plate texture as was the propensity for twinning.

For a more detailed assessment of deformation behaviour a more robust technique based on the local mechanical strain evolution at micro scale during plastic deformation is employed across various materials. Digital image correlation (DIC) and microgrid techniques in conjunction with speckle pattern analysis are documented to have been employed in this respect. Ghadbeigi et al., [10] compared DIC and microgrid methods during the in-situ tensile deformation of ferritic steels at micro level. Their work reported an overall error of 0 -34\% over the investigated regions, indicating the efficacy of the DIC method for measurements at microstructure scale. Walley et al., [11] in their work proposed using scanning electron microscope (SEM) based in-situ tests for high temperature deformation of nickel based superalloy by using hafnium oxide for the speckle particles. According to their work electron beam lithography was employed for depositing the speckle particles, whose displacements are tracked by commercial software during the deformation process. Paulsen et al., [12] in their research also compared results obtained from DIC with an etched surface against a gold speckled surface during in-situ testing of two-phased steel. The authors observed that DIC performed with gold speckles produces a spatial resolution of $0.096 \mu \mathrm{m}$ and is capable of resolving slip lines. This may be compared to the results from an etched surface which gave a spatial resolution of $2.24 \mu \mathrm{m}$ and was capable of resolving grain level local stress. Lagattu et al., [13] in their work examined the use of DIC for an in-situ SEM method which proved capable of achieving a spatial resolution of $1 \mu \mathrm{m}$ after process validation with Ti-6Al-4V. According to the authors, the method is capable of accounting for deformation processes in a range of polycrystalline materials.

Other studies have relied on the usage of grey scale secondary electron (SE) images to correlate strains at the grain level without adopting the speckle particle or micro grid approaches. Kang et al., [14] in their work utilised SEM images to correlate microscopic strain distribution and damage of dual phase steels at grain scale. Ghadbeigi et al., [15] in their work on high strength dual phase steels utilised DIC to examine localised plastic strain changes with SEM images. Tasan et al., [16] in their research also used DIC with SEM images, to differentiate between band morphologies and phase features observed in dual phase steels during in-situ tensile testing. Grey scale SE images are employed in this work for DIC correlation of images captured during the tensile deformation process.

Generally, conventional uniaxial deformation provides useful information about material mechanical properties with a global strain distribution but offers limited information on localised strain evolution especially at sheet surface defect sites. Experimental proof showing the presence and magnitude of local strain evolution at sheet defect sites and their impact on local microstructure response thus becomes important towards understanding grain level plasticity mechanisms. However, research directed at understanding the impact of surface inhomogeneity in the form of defects, notches and indents on the in-plane strain evolution at microstructural level during plastic deformation has not been extensively studied according to the knowledge of the authors. Techniques targeted at identifying the evolution of local plastic flow are of significant interest for validation of plasticity models [17]. Therefore, evaluating the strain field evolution at the phase level will aid in providing vital information on the relationship between the material microstructure and the response to deformation under various conditions. Quantitative evaluation of the localised strain evolution at the phase level for sheet at sites with and without defects will provide vital mechanistic comprehension of deformation parameters. The information provided could also be useful during material selection for sheet metal forming, processing parameters and the validation of models where incorporating defect factors into material response is key. An ex-situ technique based on SEM images and DIC of notched Ti-3Al-2.5V near alpha titanium alloy with indent surface defects, is presented in this work to ascertain their influence on the in-plane strain evolution during uniaxial tensile deformation at room temperature.

\section{Experimental procedure}

\subsection{The material}

The material researched in this work was Ti-3Al-2.5V near alpha titanium alloy with a thickness of $1.25 \mathrm{~mm}$. The alloy has reasonable cold formability coupled with intermediate strength compared to pure titanium and are mostly used for honeycomb structures and hydraulic tubing in the aerospace sector.

\subsection{Material preparation and mechanical properties}

Grinding and polishing was done on a Buehler Automet 300 pro grinder- polisher machine. For polishing, a $0.02 \mu \mathrm{m}$ cloth with $0.02 \mu \mathrm{m}$ colloidal silica and hydrogen peroxide was employed. SEM was performed on a Quanta FEG 250 microscope. The SEM micrograph of the as-received material revealed traces of $\beta$ phase (light) in $\alpha$ 
phase (dark); Figure 1a. Standard room temperature tensile testing in accordance with 1SO 6892-1:2016 was performed on the material in the three sheet-processing directions at a constant strain rate of $1 \times 10^{-3} / \mathrm{s}$. The material showed high anisotropy after room temperature testing; Figure $1 b$.
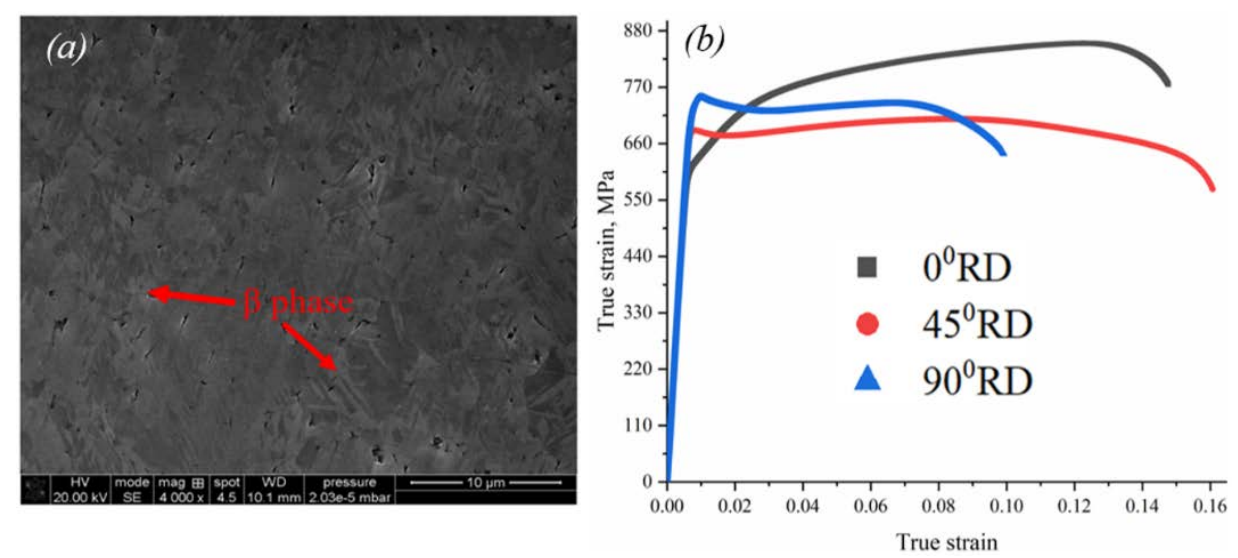

Figure 1. Ti-3Al-2.5V alloy

(a) SEM microstructure of as received material, $(b)$ tensile behaviour at room temperature

\subsection{Ex-situ uniaxial tensile test and strain analysis}

A Zwick/Roell Z150 tensile testing machine was used to deform the sample at a crosshead speed of $0.002 \mathrm{~mm} / \mathrm{s}$. A $100 \times 19 \mathrm{~mm}$ notched tensile sample with a $1 \mathrm{~mm}$ radius notch was used in this work, see Figure 2 . A notched tensile sample was chosen to ensure that regions of high stress triaxiality (yellow dotted rectangle - Figure 3) are examined for consistent tracking of the critical strain field evolution. Two HV 0.05Kg indents were created at the critical strain zones of the sample to serve as surface defects as well as a reference point for image capture during the ex-situ tensile test. Two regions of interest were examined during the tensile testing; indent defect regions (red regions) with an area of $160 \mu \mathrm{m} \times 160 \mu \mathrm{m}$ and free surfaces (blue regions) with an area of $40 \mu \mathrm{m} \times 40 \mu \mathrm{m}$, Figure 3 . The variations in magnification were accounted for during calibration of the field displacement.

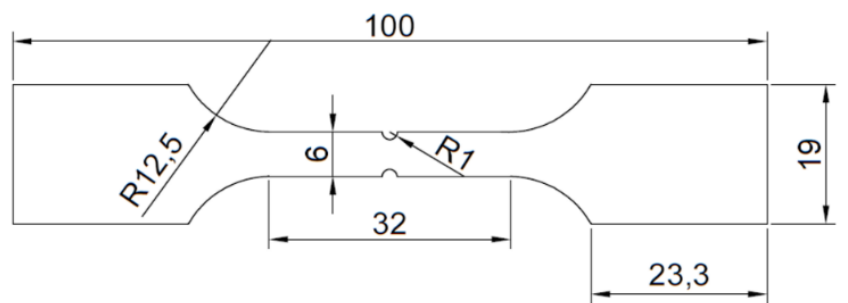

Figure 2. Test sample dimensions

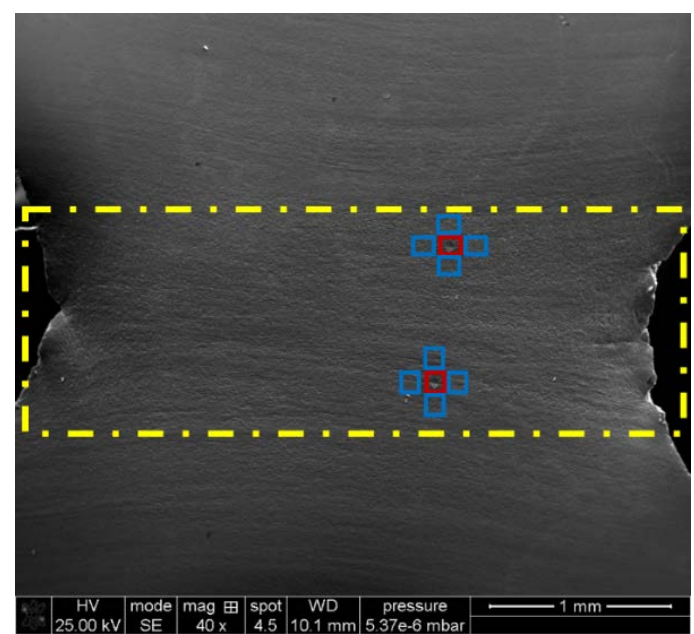

Figure 3. Zones examined during ex-situ tensile testing. Indent regions (red regions) had an area of $160 \mu \mathrm{m} \times 160 \mu \mathrm{m}$ and the free surface (blue regions) had an area of $40 \mu \mathrm{m} \times 40 \mu \mathrm{m}$ 
The tensile test was interrupted at different strain values in order to capture the microstructural evolution images during the plastic working process. A low strain rate was used to ensure that, the microstructure transformation as well as the micro damage process were sufficiently captured. The micrographs at the regions of interest were taken in SE mode. The most critical aspect of the ex-situ testing is the process of returning to and acquiring images at the same zones of interest after each image acquisition. For this, a DIC with a highly robust algorithm, capable of capturing image discontinuities must be chosen. The recorded SE microstructures for each interrupted deformation image were then loaded into Matlab based Ncorr version 1.2.2 software for the onward correlation and evaluation of field displacements and localised strain evolution at microstructure length scale.

\subsection{Digital image correlation}

The algorithm implemented in the Matlab based software, relies on the robust and computationally efficient Bing Pan's reliability-guided (RG) DIC framework [18]. The improvement of the RG-DIC approach on the traditional DIC technique is based on its ability to correct wrongly calculated points, which may occur because of roughening of the surface during deformation. Normally, these errors would be carried on during image processing resulting in incorrect computation. The RG-DIC mainly employs the zero- mean normalised cross correlation (ZNCC) coefficient as the reliability factor to compensate for such discontinuities. For a desired vector $\boldsymbol{p}$ with respect to the displacement mapping function, the ZNCC is given by [18, 19];

$$
C_{Z N C C}(\boldsymbol{p})=\frac{\sum_{x=-M}^{M} \sum_{y=-M}^{M}\left[f(x, y)-f_{m}\right] \times\left[g\left(x^{\iota}, y^{\iota}\right)-g_{m}\right]}{\sqrt{\sum_{x=-M}^{M} \sum_{y=-M}^{M}\left[f(x, y)-f_{m}\right]^{2}} \sqrt{\sum_{x=-M}^{M} \sum_{y=-M}^{M}\left[g\left(x^{\iota}, y^{\iota}\right)-g_{m}\right]^{2}}}=1-0.5 \times C_{Z N S S D}(\boldsymbol{p})
$$

ZNSSD is the zero-normalised sum of squared differences also given by;

$$
\begin{aligned}
& C_{Z N S S D}(\boldsymbol{p})=\sum_{x=-M}^{M} \sum_{y=-M}^{M}\left[\frac{f(x, y)-f_{m}}{\sqrt{\sum_{x=-M}^{M} \sum_{y=-M}^{M}\left[f(x, y)-f_{m}\right]^{2}}}-\frac{g\left(x^{\iota}, y^{\iota}\right)-g_{m}}{\sqrt{\sum_{x=-M}^{M} \sum_{y=-M}^{M}\left[g\left(x^{\iota}, y^{\iota}\right)-g_{m}\right]^{2}}}\right]^{2} \\
& f_{m}=\frac{1}{(2 M+1)^{2}} \sum_{x=-M}^{M} \sum_{y=-M}^{M}[f(x, y)] \\
& g_{m}=\frac{1}{(2 M+1)^{2}} \sum_{x=-M}^{M} \sum_{y=-M}^{M}\left[g\left(x^{\iota}, y^{\iota}\right)\right]
\end{aligned}
$$

where, $M$ is subset dimension in pixels from a reference image; $(x, y)$ is Reference subset; $\left(x^{l}, y^{l}\right)$ is target subset; $f(x, y)$ is gray level intensity at reference coordinate $(x, y)$ of reference image; $g\left(x^{l}, y^{l}\right)$ is gray level intensity at target coordinate $\left(x^{l}, y^{l}\right)$ of the target image.

This algorithm is adopted by the DIC, and directed at correlating the localised displacement of the reference image surface location together with surface locations of the target (current) images acquired during the deformation process. Micrograph features are used to trace the patterns for which correlation is attained between subsequent loading steps. Seed placements are performed for the update and to obtain good correlation with the reference image. To ensure good seed placement in this work, a strong match location for the reference image and the subsequent current image locations was enforced. A striking similarity between the reference and the transformed current subsets was obtained in this research, see Figure 4. Subsets are minute subsections of a reference image from undeformed material, which is compared against subsequent deformed (current) images by evaluation of their subsequent positions in the current configuration. Subsets enable the tracking of points on the grey scale SE images where the distinct grey scale within each subset serves as a distinct point to track that subset. Subsets are fundamentally sets of coordinate points. The cell size that is utilised for correlating the reference and current images are the subset size. For two successive subsets, the distance between them is the subset spacing.

\section{Results and discussion}

A notched tensile sample oriented transverse to the sheet rolling direction was used in this investigation. The standard force- displacement curve after each stage of deformation during the interrupted tensile testing process is shown in Figure 5. The notched sample yielded after $0.13 \mathrm{~mm}$ of travel after which a drop in load was observed. There was a continuous decrease in the load until micro-fracture nucleation sites were observed at the notch edge after a displacement of $0.54 \mathrm{~mm}$. The maximum load occurred at just about the same value of the yield point for this material when worked in the transverse direction, consistent with the stress- strain behaviour in Figure $1 b$. 

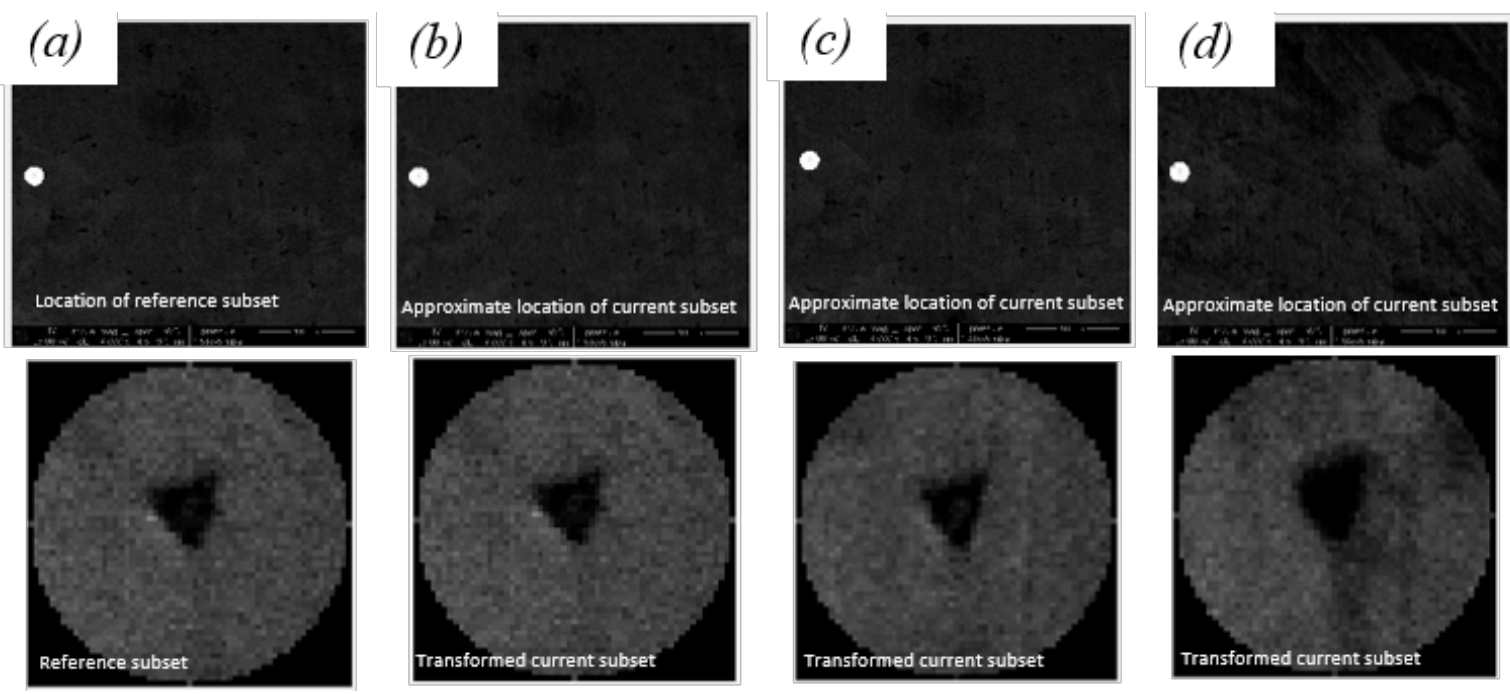

Figure 4. Seed placement matching for reference and current images

(a) Reference image (Stage 0$)$, (b) stage $0,(c)$ stage 1 , $(d)$ stage 2

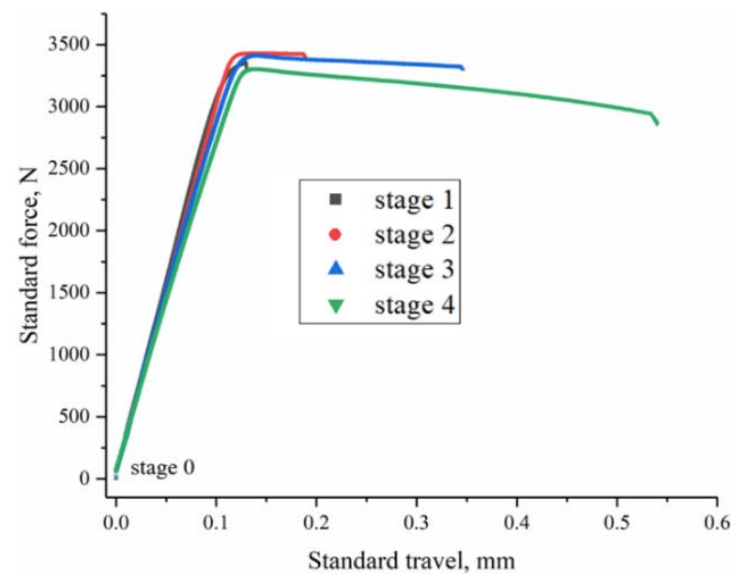

Figure 5. Standard force- travel curve for the interrupted ex-situ notch tensile deformation. The different stages of deformation (stage 0-4) correspond to micrographs labelled (a-e) respectively in Figures 6 and 9

\subsection{Localised strain evolution at free surface}

The micrographs captured during the interrupted ex-situ tensile deformation of the notched sample with their corresponding DIC localised strain maps are shown in Figure 6. The as-received microstructure (Figure 6a) and those obtained at the material yield point (Figure $6 b$ ) showed very similar microstructure features. Further deformation beyond the material yield point resulted in significant microstructure transformation, see Figure $6 c-e$. Slip lines (Figure $6 c, d$ ) were observed after the material yielded, followed by deformation bands and subsequently shear bands (Figure $6 e$ ) oriented at $45^{0}$ to the tensile axis. Slip deformation was observed very early in this material because, necking occurred abruptly as a result of the tensile strength being very close to the yield point. Slip deformation after yielding of Ti-6Al-4V was reported to evolve from single to multiple and subsequently crossslip modes during in-situ tensile deformation [20]. The deformation bands observed in Figure $6 d$ became heavily deformed following further straining resulting in roughening of the surface (Figure 6e). A match of the slip lines with the slip trace analysis graph [21] revealed that, the dominant activated deformation slip system observed in this work was prismatic slip (-1100)[11-20], with a Schmid factor of 0.46 (green line- Figure 6d). Slip trace analysis utilised in other works to ascertain the active deformation slip system present in Ti-3Al-2.5V also showed that, $80 \%$ of the 128 active slip systems examined were prismatic slip [9].

DIC analysis strain maps also revealed the main strain concentrating sites attained during the deformation process. From initial deformation up until the material yield point, the strains are seen to concentrate mostly in the softer $\beta$ phase (Figure $6 b$ ). Subsequent straining beyond the yield point resulted in the strains concentrating mostly in the regions with slip bands as well as deformation bands, Figure $6 c, d$. Further loading resulted in the formation of highly dense deformation shear bands where high strains continued to concentrate, Figure 6e. Deformation bands serving as zones of high stress concentration and acting as nucleation points for damage are reported in high strength dual phase steel [22] and ferrite- pearlite two-phase steels [12]. 

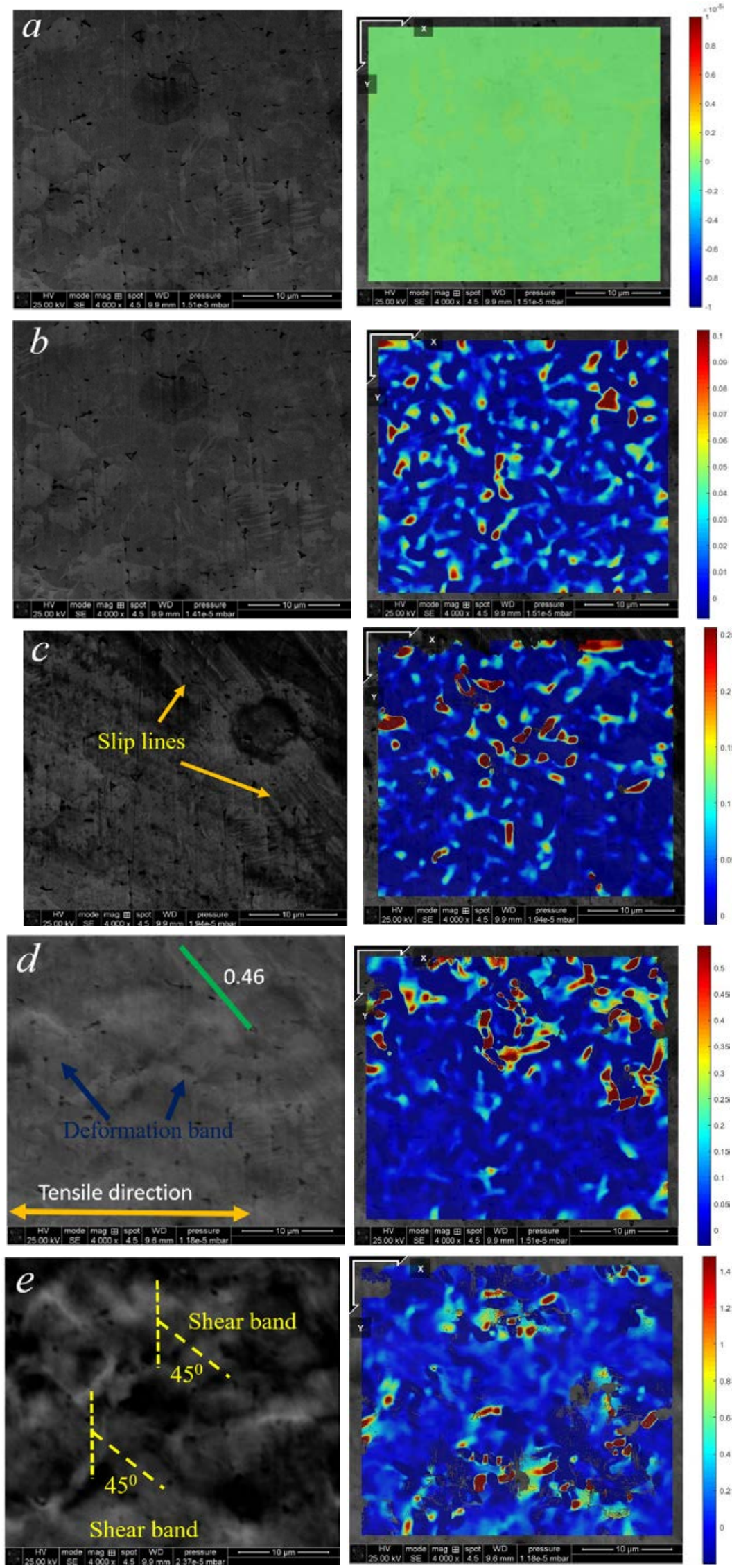

Figure 6. SEM and DIC strain maps at different stages of deformation. stage $0, \varepsilon_{\text {global }}=0 \%(b)$ stage $1, \varepsilon_{\text {global }}=$ $0.41 \%(c)$ stage $2, \varepsilon_{\text {global }}=0.58 \%(d)$ stage $3, \varepsilon_{\text {global }}=1.1 \%(e)$ stage $4 \varepsilon_{\text {global }}=1.7 \%$ 
The localised deformation strain field evolution within the phases were evaluated by identifying discrete points in the $\alpha$ and $\beta$ phases as well as the emerging deformation bands. The regions of interest chosen, with the subset radius and spacing preview for the local phase strain field evolution during the deformation process is shown in Figure 7. For a given region of interest, impoints (location of subset zones to be tracked, shown as green cross in Figure $7 b$ ) were placed in the reference image for processing and onward correlation. A subset radius of 28 and subset spacing of 1 offered the best subset tracking for the DIC in this work, Figure $7 c$.
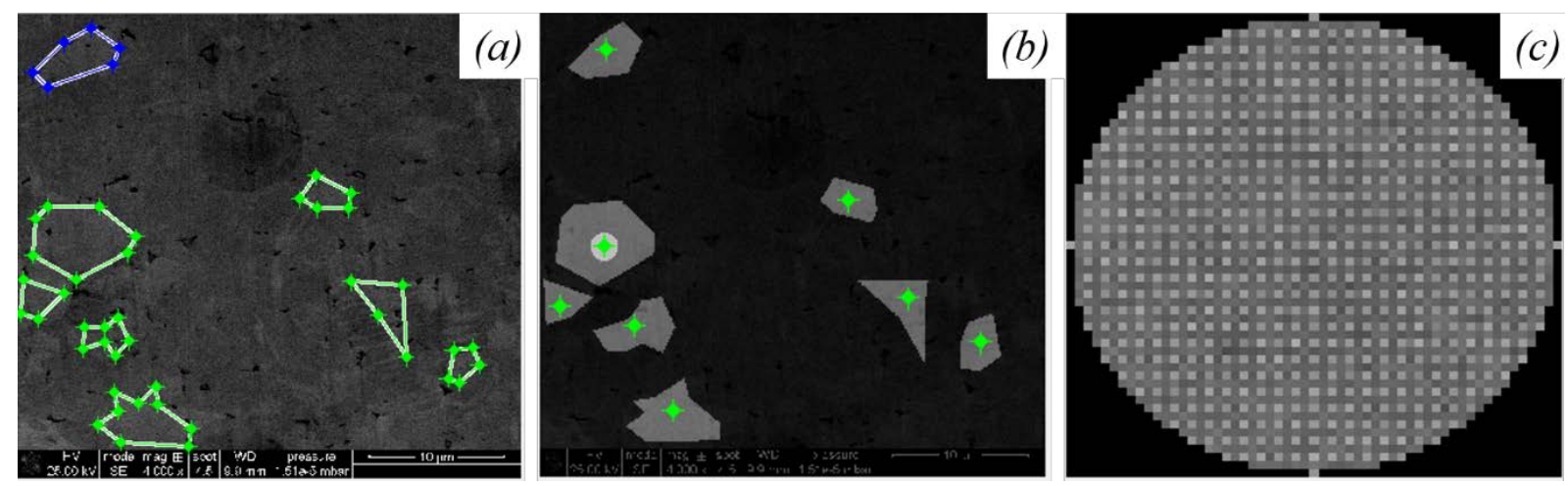

Figure 7. DIC parameters for localised phase strain evolution

(a) Regions of interest (b) Subset location, (b) subset spacing preview

A plot detailing the DIC analysis, created to quantify the phase field localised strain evolution during the deformation process is shown in Figure 8. The localised strains were highest for the corresponding global strains where deformation bands began to form. Initial straining to the yield point (global strain, $\varepsilon_{\text {global }}=0.41 \%$ ) resulted in most strain concentrating in the $\beta$ phase i.e. plastic deformation occurred mainly in the $\beta$ phase field with the localised $\alpha$ phase field remaining primarily elastic. Beyond yield both phases exhibited a similar strain distribution with slightly higher localised strains observed in the $\beta$ phase fields compared to $\alpha$ phase fields at room temperature. The maximum localised strain attained for $\alpha$ phase, $\beta$ phase and the deformation bands were $80 \%, 170 \%$ and $320 \%$ respectively at a global strain of $1.7 \%$, Figure 8 .

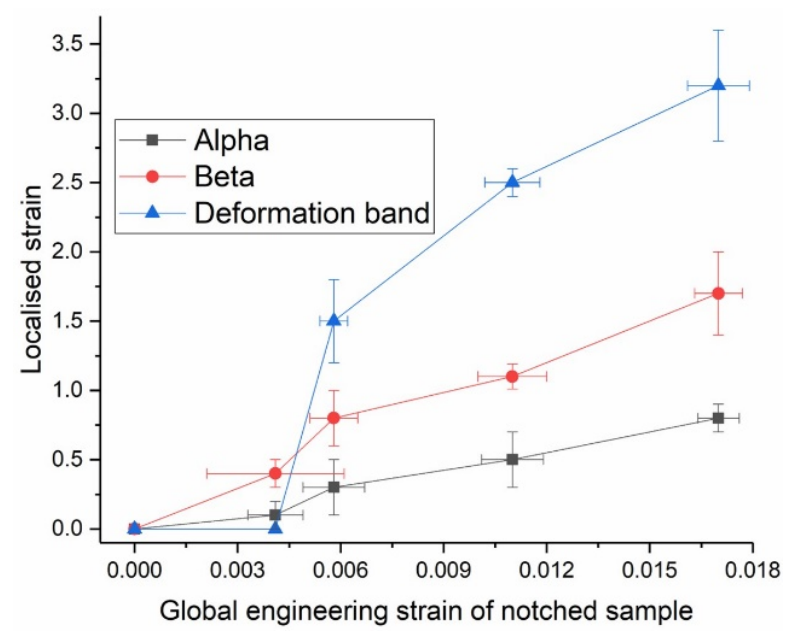

Figure 8. Localised strain evolution in $\alpha$ phase, $\beta$ phase and deformation bands

\subsection{Localised strain evolution at indent defect surface}

The micrographs obtained for the indent defect zone with their corresponding DIC strain maps are shown in Figure 9. Again, there were few differences in the micrographs between the as-received condition and those from the material yield point, Figure $9 a, b$. Deformation beyond the material yield point produced slip lines with subsequent straining resulting in the activation of shear bands (Figure $9 c, d$ ) oriented at $45^{0}$ to the tensile axis. Further straining resulted in a heavy deformation of the deformation bands near the indent edge, Figure 9e. The most outstanding feature of the deformation is the initiation and accumulation of the deformation band at the sharp tip of the defect along the tensile axis. 

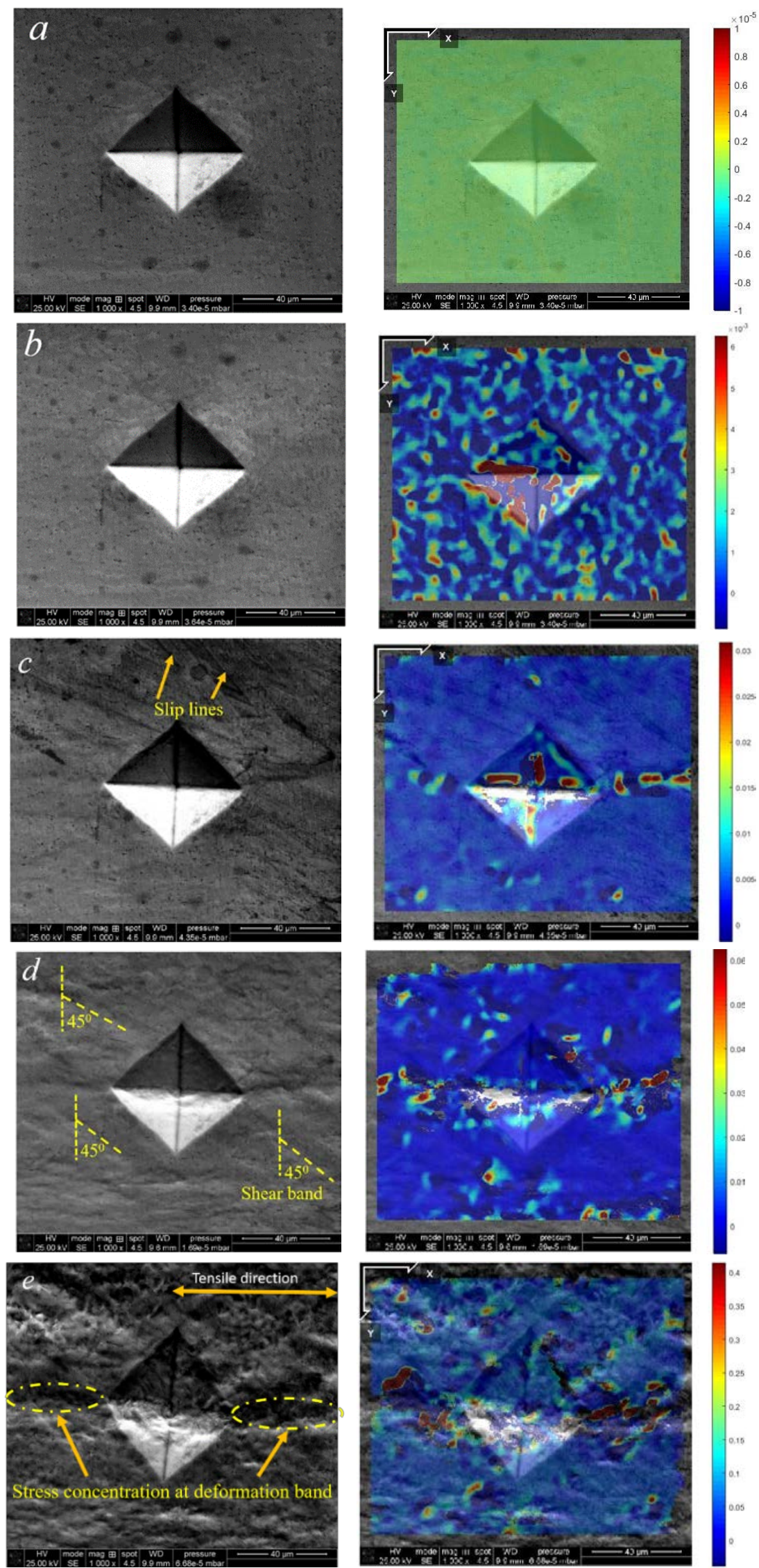

Figure 9. SEM and DIC strain maps of indent defect surface at different stages of deformation. (a) stage 0 , $\varepsilon_{\text {global }}=$ $0 \%$ (b) stage $1, \varepsilon_{\text {global }}=0.41 \%$, (c) stage $2, \varepsilon_{\text {global }}=0.58 \%(d)$ stage $3, \varepsilon_{\text {global }}=1.1 \%(e)$ stage $4, \varepsilon_{\text {global }}=1.7 \%$ 
Initial evaluation of the DIC strain map at the yield point of the indent defect region revealed strains concentrating at the defect site and at the softer $\beta$ phase field; Figure $9 b$. Further straining beyond the yield point, resulted in the strains concentrating both inside the indent defect site and at the sharp defect edge along the tensile axis; Figure $9 c$. This observation is a sharp departure from the strain concentration seen at the slip line region of the defect free surface zone, for the same loading condition; (Figure 6c). Subsequent straining resulted in increased localised strain concentration, at the shear bands and the severely strained deformation bands near the indent defect edge tip along the tensile axis; Figure $9 d$. The strains at the indent defect edge tip increased with increasing deformation load to $40 \%$ localised strain for a global strain of 1.7\%; (Figure 9e). The indent defect tip aligned to the tensile axis experienced the highest localised deformation strain as a result of high deformation band accumulation with little deformation at the indent defect edge tip oriented transverse to the tensile axis. This is probably because, the indent defect edge tip along the tensile axis was under tensile stress whereas the defect indents transverse to the tensile axis was under compressive stress. The localised strains observed at the deformation bands along the indent defect edge tip, are significantly higher than those observed in the deformed $\beta$ phase field.

For a defect free surface, the absence of artificial (defect) zones acting as high stress concentration site means strain aggregation at critical defect sites is minimised and the $\alpha$ phase field is able to sufficiently accommodate the $\beta$ phase deformation reasonably well. The presence of these indent defects serves as an obstacle to the material plastic flow by restricting the movement of dislocations and creating zones of high energy absorption resulting in the reduced values of fracture strain. The deformation bands concentrating at the tip of the indent defect act as nucleation site for early crack nucleation, propagation and growth hence the reduced localised fracture strain.

A comparative analysis was done for the localised strain evolution attained in both indent defect and free surfaces; Figure 10. Both the defect free surface and the indent defect zone exhibited higher localised strains ( $140 \%$ and $40 \%$ respectively) at the deformation band at a global strain of $1.7 \%$ showing the heterogeneous nature of the deformation observed in the material. The disparity in the localised strain evolution observed in the deformation bands and those outside the band accounts for the heterogeneity in the plastic flow. The heterogeneity could also be attributed to the formation of shear bands oriented $45^{0}$ to the tensile axis. Similar deformation trends of localised strain field distribution was observed for both sheet surface conditions over the examined global strain values.

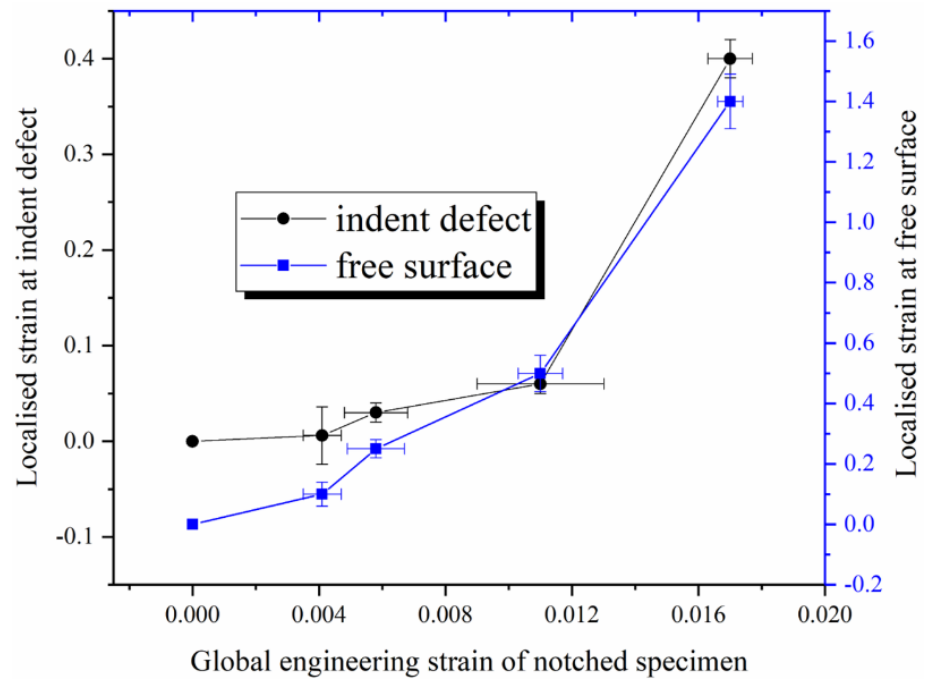

Figure 10. Localised and global strain evolution

\subsection{Damage evolution}

At global strain of $1.7 \%$, a high volume fraction of micropores with spherical morphology (size 1-3 $\mu$ m), Figure $11 b$, were observed in the mid-section of the notched tensile sample due to the high stress triaxiality [23] of the region. These micropores were observed mostly in the softer $\beta$ phase matrix. Regions where heavily deformed $\alpha$ phase field bands (relatively low) intercept the shear deformation bands (yellow dotted rectangle) produce tracks with minimised thickness, which could also promote micro crack propagation. This narrow zone of the deformation bands act as critical point where localised fracture strains are highest. Generally, because the $\alpha$ phase field experienced minimal localised deformation up to the global strain of $1.7 \%$, micro pore formation is inhibited. Microcracks initiating and propagating along phase boundaries and slip bands are observed in Ti-6Al-4V during in-situ tensile deformation [20]. These micro pores act as nucleation sites for crack coalescence and growth resulting in damage, reminiscent of ductile fracture [24, 25]. At optimum values of the acting shear stress, the micro-cracks nucleated at the notch edge are propagated along the interface with the through thickness-cracks 
oriented $45^{0}$ to the tensile axis; Figure 11a. The global damage mechanism is ductile shear fracture. Assessment of the edge crack surface shows zones with a high volume fraction of fine circular dimple texture with accompanying long tearing ridges and the presence of micro voids, which are synonymous with a ductile fracture mechanism during plastic deformation; Figure 11c. The nucleation, growth and coalescence of the micro voids results in part failure.
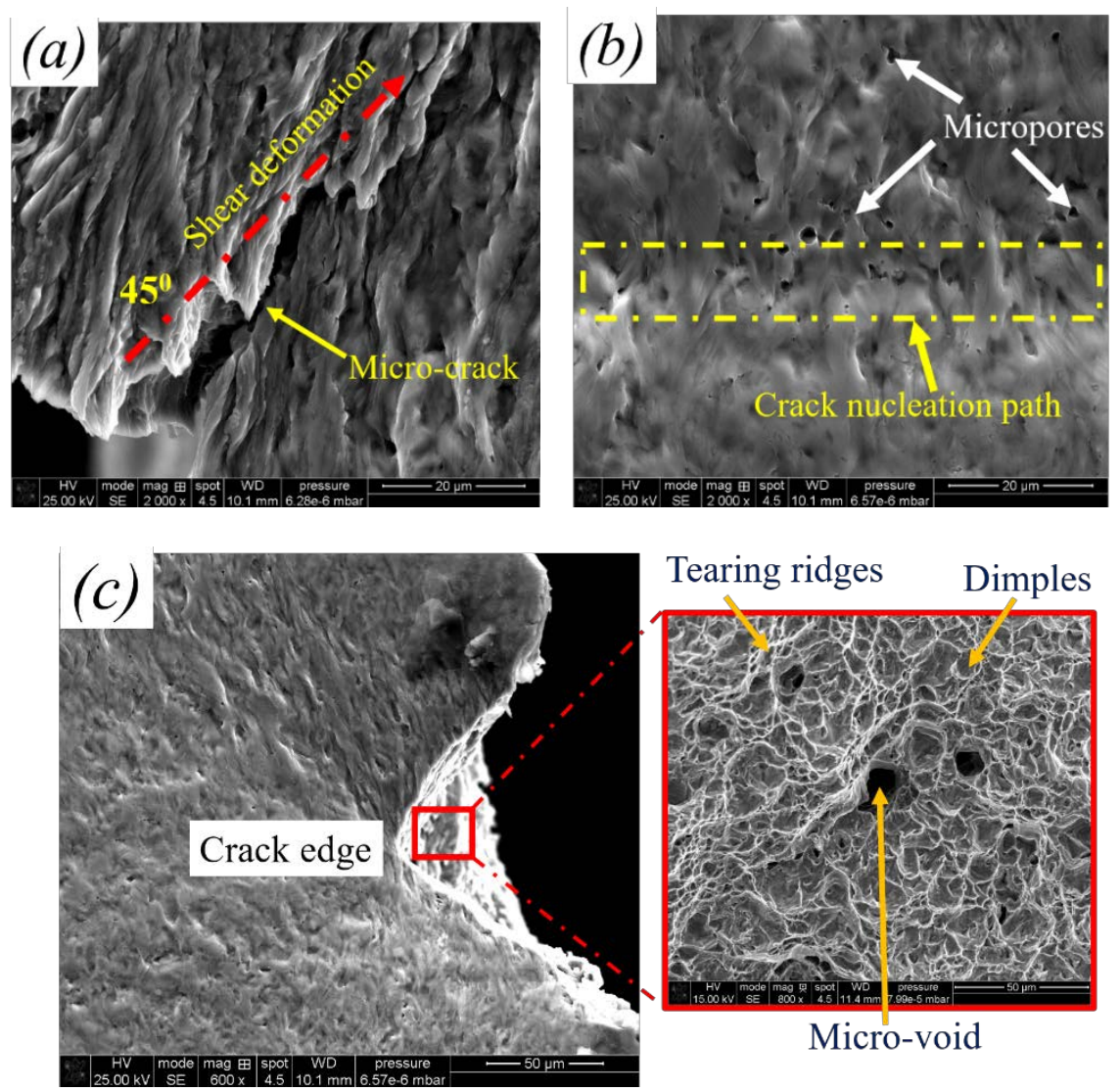

Figure 11. Damage nucleation after $1.7 \%$ global strain

(a) microcrack at notch edge, (b) micropore nucleation (c) fractograph of edge crack surface

\section{Conclusions}

Traditional uniaxial deformation provides important information about material properties with global strain distribution but offers insufficient details on localised strain evolution especially at sheet surface defect-sites such as those which may result from damage in transport and handling within the workplace. Considering the notch sensitivity of titanium and its alloys, there is the need to experimentally ascertain the magnitude of local strain evolution at sheet defect sites and their impact on global microstructure strain response during uniaxial tensile deformation. This research utilised an interrupted (ex-situ) tensile testing of a notched Ti-3Al-2.5V, scanning electron microscope and a Matlab based DIC to study the impact of sheet conditions on the localised strain evolution at room temperature. A comparative study of the performance of the material with an indented surface defect vis-à-vis a defect free surface was conducted. The research found that;

1) Slip deformation occurs abruptly due to the peculiar nature of the material when worked in the transverse direction. The tensile strength is very close to the yield point resulting in the strains initially concentrating at the slip deformation site for the free surface region.

2) The sharp edges of the indent defects act as zones of high stress concentration site. During the deformation process, the emergent localisation of deformation leads to bands which accumulate at the sharp defect edges. These bands at the tip of the indent defect pin plastic flow by acting as a nucleation site for early crack initiation, propagation and growth hence the reduced localised fracture strain limit values.

3) Overall, both the $\alpha$ and $\beta$ phases exhibited similar localised strain distribution evolution paths with slightly higher localised strains observed in the $\beta$ phase fields as compared to the $\alpha$ phase fields. This appears to be due to the $\alpha$ phase being able to sufficiently accommodate the $\beta$ phase deformation at room temperature. The maximum localised strain attained for the $\alpha$ phase, $\beta$ phase and at regions where localisation of deformation into bands 
occurred was $80 \%, 170 \%$ and $320 \%$ respectively at a global strain of $1.7 \%$. Localization of deformation into bands situated $45^{0}$ to the tensile axis formed zones of high strain concentration during the deformation process.

4) For a global strain of $1.7 \%$, the free surface exhibited higher localised fracture strain limit values (140\%) compared to the indent defect surface (40\%) showing the heterogeneous nature of the deformation observed in the material.

5) A high volume fraction of micro-pores with spherical morphology (size 1-3 $\mu \mathrm{m}$ ) are observed mostly in the softer $\beta$ phase matrix. These micro-pores act as nucleation sites for crack coalescence and growth resulting in fracture. Shear stresses acting are optimum when micro-cracks nucleated at the notch edge are oriented $45^{0}$ to the tensile axis. The damage mechanism is ductile shear fracture at a global strain of $1.7 \%$.

\section{Acknowledgement}

The authors acknowledge the support of the Advanced Forming Research Centre (AFRC) and the Department of Design, Manufacturing and Engineering Management (DMEM) of the University of Strathclyde. The authors also acknowledge Timet for material supply.

\section{References}

[1] Boyer RR. An overview on the use of titanium in the aerospace industry. Materials Science and Engineering: A. 1996;213(1-2):103-114.

[2] Jaffee RI. General physical metallurgy of titanium reviewed. JOM. 1955;7(2):247-252.

[3] Li H, Mason DE, Yang Y, Bieler TR, Crimp MA, Boehlert CJ. Comparison of the deformation behaviour of commercially pure titanium and Ti-5Al-2.5Sn (wt.\%) at 296 and 728 K. Philosophical Magazine: Part A: Material Science. 2013; 93(21):2875-2895.

[4] Lin FS, Starke EA, Chakrabortty SB, Gysler A. The effect of microstructure on the deformation modes and mechanical properties of Ti-6Al-2Nb-1Ta-0.8Mo: Part I. Widmanstätten structures. Metallurgical and Materials Transactions A. 1984;15:1229-1246.

[5] Li H, Boehlert CJ, Bierler TR, Crimp MA. Examination of the distribution of the tensile deformation systems in tension and tension-creep of Ti-6Al-4V (wt.\%) at $296 \mathrm{~K}$ and $728 \mathrm{~K}$. Philosophical Magazine: Part A: Material Science. 2015;95(7):691-729.

[6] Li H, Boehlert CJ, Bierler TR, Crimp MA. Analysis of slip activity and heterogeneous deformation in tension and tension-creep of Ti-5Al-2.5Sn (wt \%) using in-situ SEM experiments. Philosophical Magazine: Part A: Material Science. 2012;92(23):2923-2946.

[7] Fitzner A, Leo Prakash DG, Quinta da Fonseca J, Thomas M, Zhang SY, Kelleher J, Manuel P, Preuss M. The effect of aluminium on twinning in binary alpha-titanium. Acta Materialia. 2016;103:341-351.

[8] Lainé SJ, Knowles KM. $\left\{11^{-} 24\right\}$ deformation twinning in commercial purity titanium at room temperature. Philosophical Magazine: Part A: Material Science. 2015;95(20):2153-2166.

[9] Li H, Boehlert CJ, Bieler TR, Crimp MA. Analysis of the deformation behavior in tension and tension-creep of Ti-3Al-2.5V (wt pct) at $296 \mathrm{~K}$ and $728 \mathrm{~K}\left(23^{\circ} \mathrm{C}\right.$ and $\left.455^{\circ} \mathrm{C}\right)$ using in situ SEM experiments. Metallurgical and Materials Transactions A. 2014;45A(13):6053-6066.

[10] Ghadbeigi H, Pinna C, Celotto S. Quantitative strain analysis of the large deformation at the scale of microstructure: Comparison between digital image correlation and microgrid techniques. Experimental Mechanics. 2012;52:1483-1492.

[11] Walley JL, Wheeler R, Uchic MD, Mills MJ. In-situ mechanical testing for characterizing strain localization during deformation at elevated temperatures. Experimental Mechanics. 2012;52:405-416.

[12] Paulsen CO, Fagerholt E, Børvik T, Westermann I. Comparing in situ DIC results from an etched surface with a gold speckled surface. Metals. 2019;9(8):1-23.

[13] Lagattu F, Bridier F, Villechaise P, Brillaud J. In-plane strain measurements on a microscopic scale by coupling. Materials Characterization. 2006;56:10-18.

[14] Kang J, Ososkov Y, Embury JD, Wilkinson DS. Digital image correlation studies for microscopic strain distribution and damage in dual phase steels. Scripta Materialia. 2007;56(11): 999-1002.

[15] Ghadbeigi H, Pinna C, Celotto S, Yates JR. Local plastic strain evolution in a high strength dual-phase steel. Materials Science and Engineering: A. 2010;527(18-19):5026-5032.

[16] Tasan CC, Hoefnagels JPM, Geers MGD. Microstructural banding effects clarified through micrographic digital image correlation. Scripta Materialia. 2010;62(11):835-838.

[17] Pinna C, Lan Y, Kiu MF, Efthymiadis P, Lopez-Pedrosa M, Farrugia D. Assessment of crystal plasticity finite element simulations of the hot deformation of metals from local strain and orientation measurements. International Journal of Plasticity. 2015;73:24-38.

[18] Pan B. Reliability-guided digital image correlation for image. Applied optics. 2009;48(8): 1535-1542. 
[19] Pan B, Xie H, Guo Z, Hua T. Full-field strain measurement using a two dimensional Savitzky-Golay digital differentiator in digital image correlation. Optical Engineering. 2007;46(3):1-10.

[20] Zhang X, Zhang S, Zhao Q, Zhao Y, Li R, Zeng W. In-situ observations of the tensile deformation and fracture behavior of a fine-grained titanium alloy sheet. Journal of Alloys and Compounds. 2018;740:660-668.

[21] Wang H, Boehlert CJ, Wang QD, Yin DD, Ding WJ. In-situ analysis of the tensile deformation modes and anisotropy of extruded Mg-10Gd-3Y-0.5Zr (wt.\%) at elevated temperatures. International Journal of Plasticity. 2016;84:255-276.

[22] Kapp M, Hebesberger T, Kolednik OA. Micro-level strain analysis of a high-strength dual-phase steel. International Journal of Materials Research. 2011;102(6):687-691.

[23] Peng J, Wang Y, Dai Q, Liu X, Liu L, Zhang Z. Effect of stress triaxiality on plastic damage evolution and failure mode for 316L notched specimen. Metals. 2019;9(10):1-17.

[24] Samei J, Zhou L, Kang J, Wilkinson DS. Microstructural analysis of ductility and fracture in fine-grained and ultrafine-grained vanadium-added DP1300 steels. International Journal of Plasticity. 2019;117:58-70.

[25] Pineau A, Benzerga AA, Pardoen T. Failure of metals I: Brittle and ductile fracture. Acta Materialia. 2016;107:424-483.

(C) 2020 by the author(s). This work is licensed under a Creative Commons Attribution 4.0 International License (http://creativecommons.org/licenses/by/4.0/). Authors retain copyright of their work, with first publication rights granted to Tech Reviews Ltd. 